\title{
Predictive value of admission red cell distribution width-platelet ratio for no-reflow phenomenon in acute ST segment elevation myocardial infarction undergoing primary percutaneous coronary intervention
}

\author{
Turgay Celık ${ }^{1}$, Sevket Balta ${ }^{1}$, Mustafa Demır ${ }^{1}$, A. Osman Yıldırım ${ }^{1}$, \\ Mehmet Gungor Kaya ${ }^{2}$, Cengiz Ozturk ${ }^{1}$, Sait Demırkol ${ }^{1}$, \\ Murat Unlu ${ }^{1}$ Selim Kılıc ${ }^{3}$ İbrahim Aydın ${ }^{4}$, Atila Iyısoy ${ }^{1}$ \\ ${ }^{1}$ Department of Cardiology, School of Medicine, Gulhane Military Medical Academy, Etlik-Ankara, Turkey \\ ${ }^{2}$ Department of Cardiology, School of Medicine, Erciyes University, Kayseri, Turkey \\ ${ }^{3}$ Department of Epidemiology, School of Medicine, Gulhane Military Medical Academy, Etlik-Ankara, Turkey \\ ${ }^{4}$ Department of Clinical Biochemistry, School of Medicine, \\ Gulhane Military Medical Academy, Etlik-Ankara, Turkey
}

\begin{abstract}
Background: The red cell distribution width-platelet ratio (RPR), a novel inflammatory marker is currently used to predict inflammation in chronic diseases. It may be associated with adverse outcomes among artery disease but its prognostic value in ST-segment elevation myocardial infarction (STEMI) treated with primary percutaneous coronary intervention (PCI) has not been fully investigated. There is no data regarding the association between RPR and in-hospital major adverse cardiovascular events (MACEs). This study evaluated the relations between pre-procedural RPR and the in-hospital and long-term outcomes in STEMI patients undergoing primary PCI.

Methods: This study included 580 STEMI patients (77\% men, mean age: $59 \pm 12$ years). The patients were divided into two groups according to thrombolysis in myocardial infarction (TIMI) flow grades after primary PCI. No-reflow was defined as a post-PCI TIMI flow grade of 0, 1 or 2 (group 1). Angiographic success was defined as TIMI flow grade 3 (group 2).

Results: Whole blood cell count, neutrophil and lymphocyte percentages, red cell distribution width, platecrit, neutrophil-lymphocyte ratio (NLR) and RPR values were higher among patients with no-reflow. On multivariate analysis, pain to balloon time, multivessel disease, TIMI thrombus grade, tirofiban, aspirin, previous coronary artery disease, NLR, platecrit and RPR remained independent predictors of no-reflow after primary PCI. Patients in no-reflow group tended to be higher percent in-hospital MACE, including nonfatal myocardial infarction and cardiovascular mortality compared to the reflow patients.

Conclusions: Admission NLR, platecrit and RPR are independent correlates of no-reflow and in-hospital MACEs among patients with STEMI undergoing primary PCI. (Cardiol J 2016; 23, 1: 84-92)

Key words: ST segment elevation myocardial infarction, in-hospital prognosis, primary percutaneous coronary intervention, red cell distribution width-platelet ratio, platecrit, neutrophil-lymphocyte ratio
\end{abstract}

Address for correspondence: Dr Sevket Balta, Department of Cardiology, Gulhane School of Medicine, Tevfik Saglam St., 06018 Etlik-Ankara, Turkey, tel: +90 31230442 81, fax: +90 31230442 50, e-mail: drsevketb@gmail.com 


\section{Introduction}

Inflammation plays a role in the initiation and progression of the atherosclerotic process [1]. Early reperfusion with primary percutaneous coronary intervention (PCI) significantly improves the survival of patients with ST-segment elevation myocardial infarction (STEMI). Coronary artery diseases (CAD), and particularly STEMI, are the leading causes of morbidity and mortality. The "no-reflow" phenomenon manifests as an acute reduction in coronary blood flow in the absence of major epicardial coronary vessel obstruction, flow-limiting dissection, vessel spasm or thrombosis [2]. No-reflow is an important complication among patients with acute STEMI undergoing primary PCI [3]. The no-reflow phenomenon has been investigated extensively in the basic science laboratory and has entered the clinical arena. The pathophysiological mechanisms of no-reflow phenomenon have not been completely understood and its etiology appears to be multifactorial. Experimental models for no-reflow phenomenon include neutrophil accumulation, reactive oxygen species, and the coagulation cascade via endothelial dysfunction and microvascular constriction [4]. Moreover, the term "no-reflow" should be reserved for patients with thrombolysis in myocardial infarction (TIMI) grade 0 or 1 flow in the absence of other etiologies with "slow flow" referring to TIMI grade 2 flow [5]. No-reflow following primary PCI is independently associated with increased in-hospital mortality, malignant arrhythmias and cardiac failure. Persistent no-reflow may also result in post-procedural myocardial infarction (MI) or extension of MI, and is associated with a poor long-term prognosis [6].

The complete blood count (CBC) is one of the most frequently ordered laboratory tests in clinical practice. Various studies have evaluated the performance of these hematological $\mathrm{CBC}$ parameters to predict disease severity and mortality risk. Automated cell counters are routinely available in many clinical laboratories and can be used to determine red cell distrubiton width (RDW), platecrit, platelet count and and some ratios like neutrophil-lymphocyte ratio (NLR), RDW-platelet ratio (RPR). Platecrit and NLR have been known as independent predictors of impaired angiographic reperfusion and long-term mortality among STEMI patients treated with primary PCI $[7,8]$. The RPR a novel routinely available, inexpensive and easily calculated index, can predict inflammation [9]. The RPR has recently been investigated as a new predictor for major adverse cardiovascular outcomes.
There is no data, however, regarding the prognostic value of RPR in the prediction of postprocedural no-reflow and in-hospital major adverse cardiovascular events (MACEs) among STEMI patients undergoing primary PCI. Accordingly, we assessed the relationship between RPR and the no-reflow phenomenon in patients who underwent primary PCI for acute STEMI.

\section{Methods}

\section{Patients}

We examined data of consecutive 855 STEMI patients treated by primary PCI in two tertiary referral center from 2014 to 2015 . Among them, 275 patients were excluded from the study because of not undergoing primary PCI $(\mathrm{n}=75)$, missing data $(n=175)$ or ineligibility to study $(n=25)$. A total of 580 STEMI patients $(77 \%$ men, mean age: $59 \pm 12$ years) admitted within $12 \mathrm{~h}$ from symptom onset were included into the study. All the patients underwent primary PCI. The diagnosis of acute STEMI was established by using The joint European Society of Cardiology, American College of Cardiology Foundation, the American Heart Association, and the World Heart Federation (ESC/ /ACCF/AHA/WHF) committee for the definition of MI established specific electrocardiography (ECG) criteria for the diagnosis of STEMI [10]. Coronary blood flow was analyzed according to TIMI flow grade [11]. Coronary no-reflow was defined as TIMI flow grade $<3$ without clear evidence of dissection, stenosis or vasospasm [12]. Patients were classified into two groups based on postintervention TIMI flow grade [11]. No reflow was defined as TIMI flow grades $0-2$ (no-reflow group) and reflow was defined as TIMI 3 flow grade [12-15].

Patients with culprit lesion in left main coronary artery, left main stenosis $>50 \%$, previous coronary artery bypass surgery, cardiogenic shock, pain to balloon time $>12 \mathrm{~h}$, treatment with fibrinolytics in previous $24 \mathrm{~h}$, active infectious or inflammatory diseases, presence of any chronic inflammatory-autoimmune disease including rheumatologic disorders, hematalogic diseases, end-stage liver and renal failures, and known malignancy were excluded from the current study. Study protocol was approved by the local institutional Ethics Committee.

\section{Coronary intervention}

All participants underwent coronary angiography performed in multiple orthogonal projections using Judkins technique. To achieve maximal 
dilatation each coronary angiogram was preceded by intracoronary injection of $100 \mu \mathrm{g}$ nitroglycerin. All primary PCI procedures were done using standard femoral route with 6 -Fr or 7 -Fr guiding catheters. The choice of balloon predilatation, primary stenting and stent type (BMS or DES) was at discretion of treating operator. Coronary blood flow was analyzed according to TIMI flow grade. TIMI flow grades were analyzed by two different interventional cardiologists blinded to patients' clinical data. Intra and interobserver variabilities were obtained from random sample of 100 patients. Intra and interobserver variability for TIMI 0 and 1 flow grades were $4 \%$ and $6 \%$, respectively. While intra and interobserver variability for TIMI 2 flow grade were $2 \%$ and $2.4 \%$, respectively; both intraobserver and interobserver variability for TIMI 3 flow grade was $0 \%$.

Multivessel disease was defined as presence of at least 1 lesion with greater than $50 \%$ diameter stenosis in $\geq 1$ major epicardial coronary artery or its major branches remote from infarct related artery (IRA).

To evaluate clot burden we performed TIMI thrombus scale in all patients [16]. In TIMI thrombus grade 0 , no cine-angiographic characteristics of thrombus are present; in TIMI thrombus grade 1, possible thrombus is present with such angiographic characteristics as decreased contrast density, haziness, irregular lesion contour, or a smooth convex "meniscus" at the site of total occlusion suggestive but not diagnostic of thrombus; in TIMI thrombus grade 2 , there is definite thrombus, with the largest dimensions $\leq 1 / 2$ the vessel diameter; in TIMI thrombus grade 3 , there is definite thrombus but with the largest linear dimension $>1 / 2$ but $<2$ vessel diameters; in TIMI thrombus grade 4 , there is definite thrombus, with the largest dimension $\geq 2$ vessel diameters; and in TIMI thrombus grade 5 , there is total occlusion. Upon admission all the patients received aspirin $300 \mathrm{mg}$ a day, bolus intravenous unfractioned heparin 5,000 IU (70 U/kg), clopidogrel with a loading dose of $300 \mathrm{mg}$ and maintenance dose of $75 \mathrm{mg}$ and nitroglycerine. Primary stenting was performed whenever possible although balloon predilatation was used in the remaining cases. The technical aspects of the procedure, duration and pressure of inflation were determined by individual operators. Primary PCI was performed as quickly as possible (average door to first balloon time $78 \pm 14 \mathrm{~min}$ ). The use of other medications, including intravenous tirofiban $(12 \mathrm{~h})$ was left at the discretion of the attending operator (including bolus tirofiban administration).
In patients undergoing tirofiban infusion the agent was administered after primary PCI in the coronary care unit.

\section{Blood analysis and echocardiography}

Venous blood samples were drawn from antecubital veins immediately after obtaining ECG. Whole blood counting parameters were analyzed by a Sysmex K-1000 and Sysmex XN-10 Automated Hematology Analyzer (Sysmex Corporation, Kobe, Japan) autoanalyzer within 5 min of blood sampling. Whole blood sample collected in tripotassium ethylenediamineteteraacetic acid (EDTA) (7.2 mg) tubes. In all study participants transthoracic echocardiography was performed before primary $\mathrm{PCI}$ in the coronary care unit. Estimated glomerular filtration rate (eGFR) was calculated using with the Chronic Kidney Disease Epidemiology Collaboration (CKD-EPI). All echocardiographic measurements were made using commercially available devices (Vivid $3^{\circledast}$ and Vivid $7^{\circledR}$, GE Medical System, Horten, Norway) with a 3.5-MHz transducer. To evaluate left ventricular ejection fraction (LVEF) the Simpson's method was used [17].

\section{Major adverse cardiovascular events}

Major adverse cardiovascular events were defined as in-stent thrombosis, nonfatal MI, and inhospital mortality during the in-hospital follow-up period. In-stent thrombosis was defined as angiographically documented total occlusion. Non-fatal MI was defined as recurrent chest pain and/or development of new ECG changes accompanied by a new increase $\geq 20 \%$ of cardiac biomarkers measured after the recurrent event. In-hospital mortality had to be verified as death from MI, cardiac arrest, or other cardiac causes.

\section{Statistical analysis}

Continuous variables were tested for normal distribution by Kolmogorov-Smirnov test. Continuous data were expressed as mean \pm standard deviation or median (interquartile range) if not normally distributed and compared using independent samples t-test or Mann-Whitney-U tests between study groups. Categorical variables were expressed as percentages and analyzed with $\chi^{2}$ test. Differences between groups were considered significant at $\mathrm{p}<0.05$ two sided.

We investigated the effects of different variables on no reflow by calculating odds ratios (ORs) in univariate analysis for all the variables. Variables for which the unadjusted $\mathrm{p}$ value $<0.10$ in logistic 
Table 1. Baseline demographical, clinical and laboratory characteristics of the study patients.

\begin{tabular}{|c|c|c|c|}
\hline & No-reflow ( $n=198$ ) & Reflow ( $n=382$ ) & $\mathbf{P}$ \\
\hline Age [years] & $62 \pm 11$ & $58 \pm 12$ & 0.001 \\
\hline Sex (male) & $144(72.7 \%)$ & $307(80.4 \%)$ & 0.036 \\
\hline Body mass index $\left[\mathrm{kg} / \mathrm{m}^{2}\right]$ & $26.55 \pm 2.15$ & $26.35 \pm 2.25$ & 0.305 \\
\hline Previous CAD & $65(32.8 \%)$ & $70(18.3 \%)$ & $<0.001$ \\
\hline Diabetes & $72(36.4 \%)$ & $89(23.3 \%)$ & 0.001 \\
\hline Hypertension & $92(46.5 \%)$ & $155(40.6 \%)$ & 0.185 \\
\hline Smoking & $108(54.5 \%)$ & $218(57.1 \%)$ & 0.561 \\
\hline Family history & $37(18.7 \%)$ & $85(22.2 \%)$ & 0.318 \\
\hline \multicolumn{4}{|l|}{ Preprocedural medications: } \\
\hline Aspirin & $122(61.6 \%)$ & $248(64.9 \%)$ & 0.432 \\
\hline Diuretic & $25(12.6 \%)$ & $39(10.2 \%)$ & 0.378 \\
\hline BAB & $120(60.6 \%)$ & $250(65.4 \%)$ & 0.250 \\
\hline ACE-I & $133(67.2 \%)$ & $277(72.5 \%)$ & 0.180 \\
\hline Statin & $127(64.1 \%)$ & $257(67.3 \%)$ & 0.449 \\
\hline Peak CK-MB [U/L] & $102(28-144)$ & $90(24-149)$ & 0.428 \\
\hline Total cholesterol $[\mathrm{mg} / \mathrm{dL}]$ & $177(146-210)$ & $183(156-216)$ & 0.112 \\
\hline $\mathrm{TG}[\mathrm{mg} / \mathrm{dL}]$ & $117(80-158)$ & $120(78-172)$ & 0.643 \\
\hline $\mathrm{LDL}[\mathrm{mg} / \mathrm{dL}]$ & $115(90-140)$ & $116(91-140)$ & 0.785 \\
\hline $\mathrm{HDL}[\mathrm{mg} / \mathrm{dL}]$ & $37(31-44)$ & $39(34-45)$ & 0.584 \\
\hline TC/HDL ratio & $4.64(3.97-5.60)$ & $4.81(4.00-5.68)$ & 0.407 \\
\hline TG/HDL ratio & $3.08(1.97-4.84)$ & $3.08(1.87-5.00)$ & 0.933 \\
\hline Serum glucose $[\mathrm{mg} / \mathrm{dL}]$ & $149(118-210)$ & $142(114-119)$ & 0.157 \\
\hline Serum urea [mg/dL] & $40(30-52)$ & $37(29-53)$ & 0.584 \\
\hline Creatinine $[\mathrm{mg} / \mathrm{dL}]$ & $1.06(0.90-1.30)$ & $1.01(0.90-1.18)$ & 0.158 \\
\hline eGFR $\left[\mathrm{mL} / \mathrm{min} / 1.73 \mathrm{~m}^{2}\right]$ & $69(51-86)$ & $77(63-91)$ & 0.001 \\
\hline Uric acid [mg/dL] & $6.1(5.4-7.1)$ & $5.9(5.0-7.6)$ & 0.188 \\
\hline
\end{tabular}

Data are presented as mean \pm standard deviation, median (interquartile range) or $\mathrm{n}(\%)$; CAD - coronary artery disease; BAB - beta-adrenergic blocker; ACE-I — angiotensin converting enzyme inhibitor; CK-MB — creatine kinase-MB isoenzyme; TC - total cholesterol; TG - triglyceride; LDL — low density lipoprotein; HDL — high density lipoprotein; eGFR — estimated glomerular filtration rate

regression analysis were identified as potential risk markers and included in the full model. We reduced the model by using backward elimination and we eliminated potential risk markers by using likelihood ratio tests. A p value $<0.05$ was considered statistically significant two sided. Statistical analyses were performed by using SPSS 15.0 Statistical Package Program for Windows (SPSS Inc., Chicago, Illinois, USA).

\section{Results}

This study included 580 STEMI patients (77\% men, mean age: $59 \pm 12$ years). The patients were divided into two groups according to TIMI flow grades after primary PCI. The patients with TIMI flow grades $0-2$ formed no-reflow group $(\mathrm{n}=198$,
144 men, mean age: $62 \pm 11$ years) and reflow group $2(\mathrm{n}=398,307$ men, mean age: $58 \pm 12$ years), respectively. Baseline clinical, demographical and laboratory parameters were listed in Table 1. Regarding demographical parameters, patients with no-reflow were older than those with reflow ( $62 \pm 11$ vs. $58 \pm 12$ years, $p=0.001)$. Also, more men were in the reflow group $(80.4 \%$ vs. $72.7 \%$, $\mathrm{p}=0.0369)$. With respect to risk factors, there were statistically significant differences in presence of diabetes mellitus $(\mathrm{p}=0.001)$ and previous CAD $(\mathrm{p}<0.001)$ as shown in Table 1 . Besides, eGFR values were lower in patients with no-reflow compared to those in reflow patients (69 vs. 77 , $\mathrm{p}=0.001)$.

The comparison of angiographic and echocardiographic characteristics of the two groups showed 
Table 2. Angiographic and echocardiographic characteristics of the study patients.

\begin{tabular}{|c|c|c|c|}
\hline & No-reflow $(n=198)$ & Reflow $(n=382)$ & $\mathbf{P}$ \\
\hline Pain to balloon time [h] & $6.0(5.0-6.5)$ & $5.5(4.0-6.0)$ & $<0.001$ \\
\hline Tirofiban use & $67(33.8 \%)$ & $83(21.7 \%)$ & 0.002 \\
\hline Admission LVEF [\%] & $46.0 \pm 9.5$ & $48.6 \pm 8.2$ & 0.001 \\
\hline TIMI thrombus grade: & & & 0.006 \\
\hline Grade 0 & $5(25 \%)$ & $26(6.8 \%)$ & \\
\hline Grade 1 & $10(5.1 \%)$ & $20(5.2 \%)$ & \\
\hline Grade 2 & $21(10.6 \%)$ & $38(9.9 \%)$ & \\
\hline Grade 3 & $29(14.6 \%)$ & $90(23.6 \%)$ & \\
\hline Grade 4 & $46(23.2 \%)$ & $54(14.1 \%)$ & \\
\hline Grade 5 & $87(43.9 \%)$ & $154(40.3 \%)$ & \\
\hline Number of narrowed vessel: & & & 0.001 \\
\hline Single vessel & $59(29.8 \%)$ & $205(53.7 \%)$ & \\
\hline Multivessel & $139(70.2 \%)$ & $177(46.3 \%)$ & \\
\hline Infarct related artery: & & & 0.823 \\
\hline LAD & $91(46.0 \%)$ & $179(46.9 \%)$ & \\
\hline CFX & $38(19.2 \%)$ & $79(20.7 \%)$ & \\
\hline RCA & $69(34.8 \%)$ & $124(32.5 \%)$ & \\
\hline \multicolumn{4}{|l|}{$\mathrm{PCl}$ procedure: } \\
\hline Balloon angioplasty & $15(7.6 \%)$ & $20(5.2 \%)$ & 0.745 \\
\hline Balloon + stenting & $147(74.2 \%)$ & $272(71.3 \%)$ & 0.320 \\
\hline Primary stenting & $36(18.2 \%)$ & $90(23.5 \%)$ & 0.295 \\
\hline Stent type: & & & 0.125 \\
\hline BMS & $169(85.4 \%)$ & $315(82.5 \%)$ & \\
\hline DES & $14(7.1 \%)$ & $47(12.3 \%)$ & \\
\hline Final balloon pressure [atm] & $16.15 \pm 2.46$ & $16.43 \pm 2.59$ & 0.224 \\
\hline Stent length [mm] & $18.08 \pm 4.69$ & $17.86 \pm 3.89$ & 0.147 \\
\hline Stent diameter $[\mathrm{mm}]$ & $3.20 \pm 0.39$ & $3.14 \pm 0.40$ & 0.620 \\
\hline
\end{tabular}

Data are presented as mean \pm standard deviation, median (interquartile range) or $\mathrm{n}(\%)$; LVEF — left ventricular ejection fraction; TIMI thrombolysis in myocardial infarction; LAD - left anterior descending artery; CFX - circumflex coronary artery; RCA — right coronary artery; $\mathrm{PCl}$ - percutaneous coronary intervention; BMS — bare metal stent; DES - drug eluting stent

no statistically significant differences apart from chest pain to balloon time, admission LVEF values, TIMI thrombus grade, tirofiban use, and presence of multivessel disease (Table 2). Pain to balloon time in no-reflow group was longer than that of the patients in reflow group as presented in Table 2 (6.0 vs. $5.5 \mathrm{~h}, \mathrm{p}<0.001)$. Also no-reflow group had higher TIMI thrombus grades and tirofiban use (Table $2 ; \mathrm{p}<0.006$ and $\mathrm{p}=0.002$, respectively). There was more patients with multivessel disease in no-reflow group compared to that of reflow group (Table $2 ; 70.2 \%$ vs. $46.3 \%, \mathrm{p}=0.001$ ). The left anterior descending coronary artery was the most common IRA in both study groups. Percent stent implantations and type of stents were similar among groups. Admission LVEF was lower in the no-reflow group compared to those of reflow group as shown in Table $2(46.0 \pm 9.5 \%$ vs. $48.6 \pm 8.2 \%$, $\mathrm{p}<0.001)$.

The comparison of admission hematological parameters of both groups were presented in Table 3 . According to admission whole blood cell (WBC) count results, the patients in no-reflow group had significantly higher WBC count, neutrophil and lymphocyte percentages, RDW, platecrit, NLR and RPR (Fig. 1) when compared to those in reflow patients (Table 3 ).

Effects of some clinically important variables on the no-reflow were analyzed by using univariate and multivariate logistic regression analyses. Data for two groups were combined and all the variables were analyzed in univariate analysis as 
Table 3. Comparison of hematological parameters of the study groups.

\begin{tabular}{lccc}
\hline & No-reflow $(\mathbf{n}=\mathbf{1 9 8})$ & Reflow $(\mathbf{n}=382)$ & P \\
\hline White blood cell $\left[\times 10^{9} / \mathrm{L}\right]$ & $11.7(9-15)$ & $11.0(9-13)$ & 0.088 \\
Neutrophil [\%] & $64.15(47-81)$ & $59.14(43-72)$ & 0.001 \\
Lymphocyte [\%] & $16.85(11-24)$ & $18.69(12-26)$ & 0.017 \\
Eosinophil [\%] & $1.36(1-1.9)$ & $1.54(1-2.4)$ & 0.158 \\
Basophil [\%] & $0.60(0.3-0.9)$ & $0.64(0.3-0.7)$ & 0.705 \\
Monocyte [\%] & $6.45(5.0-8.1)$ & $7.16(5.6-8.9)$ & 0.146 \\
Neutrophil-lymphocyte ratio & $4.11(3-5.3)$ & $2.80(2-4.37)$ & $<0.001$ \\
Hemoglobin [g/dL] & $14.00 \pm 1.78$ & $14.30 \pm 1.98$ & 0.100 \\
RDW [\%] & $13.83 \pm 1.44$ & $13.39 \pm 1.42$ & $<0.001$ \\
Platelet count [×10\%] & $238(190-289)$ & $246(200-287)$ & 0.285 \\
Platecrit & $0.23(0.19-0.30)$ & $0.21(0.18-0.25)$ & 0.003 \\
Platelet-lymphocyte ratio & $130(91-180)$ & $118(82-179)$ & 0.280 \\
RDW-platelet ratio & $5.68(4.79-7.39)$ & $5.38(4.55-6.67)$ & 0.016 \\
\hline
\end{tabular}

Data are presented as mean \pm standard deviation or median (interquartile range); RDW — red cell distribution width

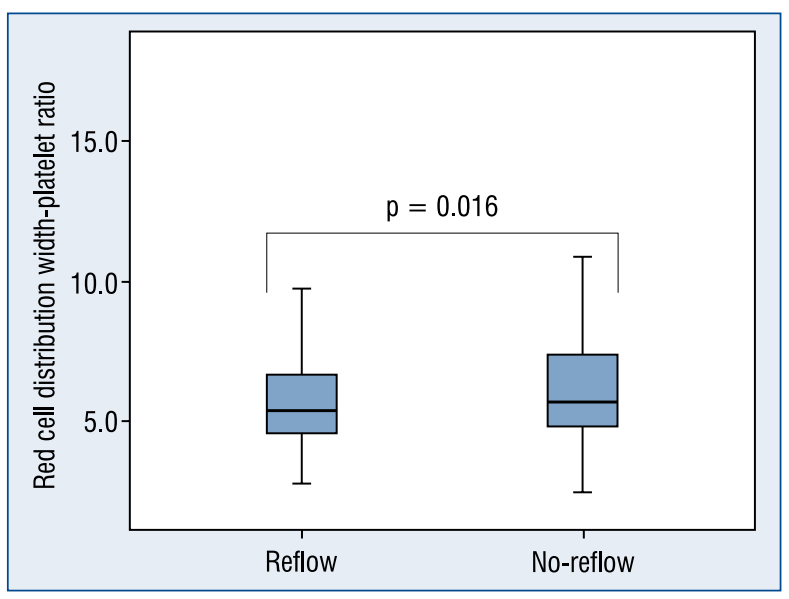

Figure 1. Median red cell distribution width to platelet ratios were statistically significantly higher in patients with no-reflow compared to those of the patients with reflow.

the predictor of no-reflow as shown in Table 4. Some variables associated with impaired flow after primary PCI were significantly different between two study groups. Independent contributions age, male gender, diabetes mellitus, LVEF on admission, eGFR, pain to balloon time, multivessel disease, TIMI thrombus grade, tirofiban, aspirin, previous CAD, NLR, platecrit and RPR were analyzed in multivariate backward logistic regression (Table 4). On multivariate analysis, pain to balloon time, multivessel disease, TIMI thrombus grade, tirofiban, aspirin, previous CAD, NLR, platecrit and
RPR remained independent predictors of no reflow after primary PCI. Adjusted ORs were calculated as 1.52 for pain to balloon time $(\mathrm{p}<0.001$; $\mathrm{CI}=1.19$ -1.81 ), 2.63 for multivessel disease $(\mathrm{p}<0.001$; $\mathrm{CI}=1.29-1.81), 1.59$ for TIMI thrombus grade $(\mathrm{p}<0.001 ; \mathrm{CI}=1.32-1.92), 0.30$ for tirofiban $(\mathrm{p}<0.001 ; \mathrm{CI}=0.19-0.49), 0.41$ for aspirin $(\mathrm{p}=0.003$; $\mathrm{CI}=0.22-0.73), 2.54$ for previous $\mathrm{CAD}$ $(\mathrm{p}=0.001 ; \mathrm{CI}=1.49-4.33), 1.07$ for NLR $(\mathrm{p}=0.042$; $\mathrm{CI}=1.01-1.14), 1.23$ for platecrit $(\mathrm{p}<0.001$; $\mathrm{CI}=1.16-1.30)$ and 1.87 for RPR $(\mathrm{p}<0.001$; $\mathrm{CI}=1.54-2.26)$ as shown in Table 4.

In-hospital MACEs of the study patients were given in Table 5. Patients in no-reflow group tended to be higher percent in-hospital MACE, including nonfatal $\mathrm{MI}$ and cardiovascular mortality compared to those of reflow patients $(\mathrm{p}<0.001$ for all parameters).

\section{Discussion}

The present study demonstrated that WBC count, neutrophil and lymphocyte percentages, RDW, platecrit, NLR and RPR values were higher among patients with no-reflow.

Due to the growing understanding in recent years of the role of inflammatory status in the initiation and progression of atherosclerosis, epidemiological studies have focused on markers of the inflammatory status and its relation to adverse outcomes in patients with different CAD phenotypes. In patients with STEMI undergoing coronary angiography, short-term mortality risk 
Table 4. Effects of various variables on no reflow in univariate and multivariate logistic regression analyses.

\begin{tabular}{lcccccc}
\hline & Unadjusted OR & $95 \% \mathbf{C l}$ & $\mathbf{P}$ & Adjusted OR & $95 \% \mathbf{C l}$ & $\mathbf{P}$ \\
\hline Age & 1.03 & $1.01-1.04$ & $<0.001$ & 1.01 & $0.99-1.03$ & 0.300 \\
Gender (male) & 1.54 & $1.03-2.29$ & 0.037 & 1.16 & $0.69-1.95$ & 0.572 \\
Beta-blocker & 0.81 & $0.57-1.15$ & 0.251 & & & \\
Hypertension & 1.27 & $0.89-1.79$ & 0.174 & & & \\
Diabetes & 1.88 & $1.29-2.74$ & 0.001 & 1.34 & $0.81-2.22$ & 0.251 \\
LVEF & 0.97 & $0.95-0.99$ & 0.001 & 0.99 & $0.96-1.01$ & 0.365 \\
Smoking & 0.90 & $0.63-1.27$ & 0.562 & & & \\
Body mass index & 1.04 & $0.96-1.12$ & 0.304 & & & \\
eGFR & 0.99 & $0.99-1.00$ & 0.295 & 1.00 & $0.99-1.01$ & 0.221 \\
Stent length & 1.00 & $0.97-1.04$ & 0.619 & & & \\
Stent diameter & 0.95 & $0.89-2.16$ & 0.148 & & & \\
Pain to balloon time & 1.52 & $1.32-1.74$ & $<0.001$ & 1.52 & $1.29-1.81$ & $<0.001$ \\
Multivessel disease & 2.73 & $1.89-3.93$ & $<0.001$ & 2.63 & $1.68-4.11$ & $<0.001$ \\
TIMl thrombus grade & 1.15 & $1.02-1.29$ & 0.027 & 1.59 & $1.32-1.92$ & $<0.001$ \\
Tirofiban & 0.54 & $0.37-0.80$ & 0.002 & 0.30 & $0.19-0.49$ & $<0.001$ \\
Aspirin & 0.87 & $0.61-1.24$ & 0.430 & 0.41 & $0.22-0.73$ & 0.003 \\
Previous CAD & 2.18 & $1.47-3.23$ & $<0.001$ & 2.54 & $1.49-4.33$ & 0.001 \\
NLR & 1.11 & $1.04-1.17$ & 0.001 & 1.07 & $1.01-1.14$ & 0.042 \\
Platecrit & 1.04 & $1.02-1.07$ & 0.002 & 1.23 & $1.16-1.30$ & $<0.001$ \\
RDW-platelet ratio & 1.12 & $1.02-1.23$ & 0.022 & 1.87 & $1.54-2.26$ & $<0.001$ \\
\hline
\end{tabular}

${ }^{*}$ Adjusted for age, gender, diabetes, LVEF on admission, eGFR, pain to balloon time, multivessel disease, TIMI thrombus grade, tirofiban, aspirin, previous CAD, NLR, platecrit and RDW-platelet ratio; OR - odd ratio; CI - confidence interval; LVEF - left ventricular ejection fraction; eGFR - estimated glomerular filtration rate; TIMI - thrombolysis in myocardial infarction; CAD - coronary artery disaese; NLR - neutrophil-lymphocyte ratio; RDW — red cell distribution width

Table 5. In-hospital major adverse cardiac events of study groups.

\begin{tabular}{lccc}
\hline & No-reflow $(\mathbf{n}=\mathbf{1 9 8})$ & Reflow $(\mathbf{n}=382)$ & $\mathbf{P}$ \\
\hline In-hospital MACEs: & $55(27.8 \%)$ & $31(8.1 \%)$ & $<0.001$ \\
In-stent thrombosis & $10(5.1 \%)$ & $13(3.4 \%)$ & 0.335 \\
Non-fatal myocardial infarction & $30(15.2 \%)$ & $13(3.4 \%)$ & $<0.001$ \\
In-hospital mortality & $46(23.2 \%)$ & $12(3.1 \%)$ & $<0.001$ \\
\hline
\end{tabular}

Data are presented as mean \pm standard deviation, median (interquartile range) or $\mathrm{n}(\%)$; MACE — major adverse cardiac events

displays a considerable variability. Early and individualized risk stratification in each patient allows for a more precise decision-making with regard to the choice of pharmacologic and interventional treatments, allocation of clinical resources and triage among alternative levels of hospital care. To make such kind of planning, one must be aware of the risk factors related to morbidity and mortality in STEMI patients undergoing angiography. One of the early late mortality factors as the no-reflow phenomenon may be defined as incomplete reper- fusion at the microvascular level despite adequate recanalization of the occluded artery. The existence of no-reflow phenomenon was initially debated; however, this pathological process can be accelerated by coronary reperfusion, which gives rise to tissue edema, endothelial injury, plugging of capillaries by neutrophils and microthrombi, and inflammation due to generation of free radicals and complement activation [18]. Several studies have shown a relationship between no-reflow phenomenon and increased inflammatory activity. Recent 
studies have shown that no-reflow phenomenon is associated with a higher prevalence of early and late morbidity and mortality $[3,19,20]$. The CBC is one of the most frequently ordered laboratory tests in clinical practice. CBC is the most widely available laboratory data in early in-hospital period, universally available in the first $30 \mathrm{~min}$. CBC is an easy, inexpensive, routine examination technique that give us information about the blood contents; the red and white cells, platelets, the counts and dimensions of subgroups of cells, and parameters like the distribution widths, some ratios. Various studies have evaluated the performance of these hematological $\mathrm{CBC}$ parameters to predict disease severity and mortality risk of STEMI. For example, platelets play a central role in the pathophysiology of an acute MI by contributing to the thrombotic occlusion of the IRA [21]. In addition to platelet count, platecrit is also inexpensive and easily available marker, which provides complete information on total platelet mass. High platecrit values on admission are independently associated with long-term adverse outcomes in patients with STEMI who undergo primary angioplasty [7]. Also, NLR has received attention due to its role as an independent prognostic factor for CAD. Many studies have investigated the relation between NLR and the short-term morbidity and mortality in patient with STEMI undergoing primary PCI. The NLR, an inexpensive and easily measurable laboratory variable, is independently associated with the development of no-reflow and in-hospital MACEs in patients with STEMI undergoing primary PCI [22]. We have reported same results in present study. An elevated RDW has been reported to be associated with mortality and other severe adverse outcomes in patients with STEMI who undergo primary angioplasty [23]. RDW is a widely available, easily derived and reproducible marker of inflammation. Increased RDW, independent of hemoglobin values, have been demonstrated to be associated with negative clinical outcomes in patients with heart failure, previous MI, stable $\mathrm{CAD}$, strokes, infections and peripheral artery disease [24]. The RDW was increased in patients and was positively correlated with bilirubin, creatinine levels, prothrombin time, and negatively correlated with platelet count and albumin level. The half-life of red blood cells is higher than bilirubin and albumin; therefore, the RDW represented a more stable index. RPR, a novel marker is a more powerful predictive index of significant fibrosis but is equally powerful in the prediction of fibrosis [23]. The RPR reflects inflammation severity. RPR is used in clinical practice with the accompanying assessments, it could be a useful and important marker for predicting the mortality of patients with some chronic diseases [25]. RPR, a cheap, routinely available and easily calculated index, can predict inflammation in patients with no-reflow when considering the pathophysiology of no-reflow phenomenon in patients undergoing primary PCI. In context, we have considered that RPR levels may be associated with no-reflow phenomenon. RPR levels are higher in no-reflow phenomenon compared with reflow groups. Also, we reported that RPR levels are independent predictors of no-reflow after primary PCI. So, RPR is considered as a novel inflammatory marker in patients with STEMI.

\section{Limitations of this study}

The main limitation of our study is the relatively small sample size. Because high-sensitivity $\mathrm{C}$-reactive protein is not evaluated routinely in patients undergoing elective coronary angiography in our department, this test was not included to compare inflammatory status between the groups. Furthermore, we did not analyze the causes of elevated RDW values, such as iron, folic acid or vitamin B12 deficiency. Finally, we excluded some diseases that may influence RPR levels; however some diseases may be unrecognized in our study group.

\section{Conclusions}

Admission RPR levels are simple, easily obtained inflammatory marker in no-reflow and in-hospital MACEs among STEMI patients undergoing primary PCI. Therefore, the predictive performance of the RPR should be further confirmed in multi-center, prospectively designed studies.

\section{Conflict of interest: None declared}

\section{References}

1. Balta S, Celik T, Mikhailidis DP et al. The relation between atherosclerosis and theneutrophil-lymphocyte ratio. Clin Appl Thromb Hemost, 2015 Feb 9; In press.

2. Durante A, Camici PG. Novel insights into an "old" phenomenon: The no reflow. Int J Cardiol, 2015; 187: 273-280.

3. Celik T, Kaya MG, Akpek M et al. Predictive value of admission platelet volume indices for in-hospital major adverse cardiovascular events in acute ST-segment elevation myocardial infarction. Angiology, 2015; 66: 155-162.

4. Schwartz BG, Kloner RA. Coronary no reflow. J Mol Cell Cardiol, 2012; 52: 873-882. 
5. The Thrombolysis in Myocardial Infarction (TIMI) trial. Phase I findings. TIMI Study Group. N Engl J Med, 1985; 312: 932-936.

6. Mehta RH, Harjai KJ, Boura J et al. Prognostic significance of transient no-reflow during primary percutaneous coronary intervention for ST-elevation acute myocardial infarction. Am J Cardiol, 2003; 92: 1445-1447.

7. Uğur M, Ayhan E, Bozbay $\mathrm{M}$ et al. The independent association of plateletcrit with long-term outcomes in patients undergoing primary percutaneous coronary intervention. J Crit Care, 2014; 29: 978-981.

8. Sen N, Afsar B, Ozcan F et al. The neutrophil to lymphocyte ratio was associated with impaired myocardial perfusion and long term adverse outcome in patients with ST-elevated myocardial infarction undergoing primary coronary intervention. Atherosclerosis, 2013; 228: 203-210.

9. Taefi A, Huang C-C, Kolli K, Ebrahimi S, Patel M. Red cell distribution width to platelet ratio, a useful indicator of liver fibrosis in chronic hepatitis patients. Hepatol Int, 2015; 9: 454-460.

10. Thygesen K, Alpert JS, Jaffe AS et al. Third universal definition of myocardial infarction. Circulation, 2012; 126: 2020-2035.

11. The Thrombolysis in Myocardial Infarction (TIMI) trial. Phase I findings. TIMI Study Group. N Engl J Med, 1985; 312: 932-936.

12. Rezkalla SH, Kloner RA. No-reflow phenomenon. Circulation, 2002; 105: 656-662.

13. Rezkalla SH, Dharmashankar KC, Abdalrahman IB, Kloner RA. No-reflow phenomenon following percutaneous coronary intervention for acute myocardial infarction: Incidence, outcome, and effect of pharmacologic therapy. J Interv Cardiol, 2010; 23: 429-436.

14. Ito H. No-reflow phenomenon and prognosis in patients with acute myocardial infarction. Nat Clin Pract Cardiovasc Med, 2006; 3: 499-506.

15. Eeckhout E, Kern MJ. The coronary no-reflow phenomenon: A review of mechanisms and therapies. Eur Heart J, 2001; 22: 729-739.
16. Gibson CM, de Lemos JA, Murphy SA et al. Combination therapy with abciximab reduces angiographically evident thrombus in acute myocardial infarction: A TIMI 14 substudy. Circulation, 2001; 103: 2550-2554.

17. Lang RM, Bierig M, Devereux RB et al. Recommendations for chamber quantification. Eur J Echocardiogr, 2006; 7: 79-108.

18. Niccoli G, Burzotta F, Galiuto L, Crea F. Myocardial no-reflow in humans. J Am Coll Cardiol, 2009; 54: 281-292.

19. Kurtul A, Murat SN, Yarlioglues M, Duran M, Celik IE, Kilic A. Mild to moderate renal impairment is associated with no-reflow phenomenon after primary percutaneous coronary intervention in acute myocardial infarction. Angiology, 2015; 66: 644-651.

20. Ndrepepa G, Tiroch K, Fusaro M et al. 5-year prognostic value of no-reflow phenomenon after percutaneous coronary intervention in patients with acute myocardial infarction. J Am Coll Cardiol, 2010; 55: 2383-2389.

21. Martin JF, Plumb J, Kilbey RS, Kishk YT. Changes in volume and density of platelets in myocardial infarction. Br Med J (Clin Res Ed), 1983; 287: 456-459.

22. Akpek M, Kaya MG, Lam YY et al. Relation of neutrophil/lymphocyte ratio to coronary flow to in-hospital major adverse cardiac events in patients with ST-elevated myocardial infarction undergoing primary coronary intervention. Am J Cardiol, 2012; 110: 621-627.

23. Chen B, Ye B, Zhang J, Ying L, Chen Y. RDW to platelet ratio: A novel noninvasive index for predicting hepatic fibrosis and cirrhosis in chronic hepatitis B. PLoS One, 2013; 8: e68780.

24. Sun X, Chen W, Sun Z et al. Impact of red blood cell distribution width on long-term mortality in patients with ST-elevation myocardial infarction. Cardiology, 2014; 128: 343-348.

25. Cetinkaya E, Senol K, Saylam B, Tez M. Red cell distribution width to platelet ratio: New and promising prognostic marker in acute pancreatitis. World J Gastroenterol, 2014; 20: 14450-14454 . 